\title{
Accelerating the Training Process of Generative Query Network
}

\author{
Wenbing Zhang \\ Tianjian Yang \\ wenbing2019@iscas.cn.cn \\ yangtianjian19@mails.ucas.ac.cn \\ Institute of Software, Chinese Academy of Sciences \\ China \\ University of Chinese Academy of Sciences \\ Beijing, China \\ Guiyang Academy of Information Technology \\ Guiyang, China \\ Feng Chen* \\ Qiao Sun* \\ chenfeng@iscas.ac.cn \\ sunqiao@iscas.ac.cn \\ Institute of Software, Chinese Academy of Sciences \\ Beijing, China \\ Guiyang Academy of Information Technology \\ Guiyang, China
}

\author{
Kohei Takeda \\ Kohei.Takeda@nttdata.com \\ NTT DATA Corporation \\ Tokyo, Japan
}

\author{
Tomoya Kaburaki \\ Tomoya.Kaburaki@nttdata.com \\ NTT DATA Corporation \\ Tokyo, Japan
}

\begin{abstract}
GQN (Generative Query Network) is a novel unsupervised generative model which is intended for neural rendering tasks. However, the time-consuming training process makes it difficult to deploy GQN. In this paper, we propose two methods to boost its training speed. Computationally, a two-phased method is proposed so that the training time is greatly reduced due to a much smaller parameter set without significant loss of image quality. We also adopt the gradient accumulation technique and avoid redundant communication. Experiments are carried out on a typical dense GPGPU platform and the result shows that, remaining similar image quality, our two-phase training approach can reduce $56.92 \%$ training time and the gradient accumulation gains $9.86 \%$ speedup.
\end{abstract}

\section{CCS CONCEPTS}

- Computing methodologies $\rightarrow$ Parallel algorithms.

\section{KEYWORDS}

GQN training acceleration, neural rendering

\section{ACM Reference Format:}

Wenbing Zhang, Tianjian Yang, Kohei Takeda, Feng Chen, Qiao Sun, and Tomoya Kaburaki. 2021. Accelerating the Training Process of Generative Query Network. In 2021 2nd International Conference on Computing, Networks and

${ }^{*}$ Both are corresponding authors.

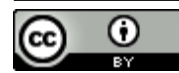

This work is licensed under a Creative Commons Attribution International 4.0 License.

CNIOT2021, May 20-22, 2021, Beijing, China

(c) 2021 Copyright held by the owner/author(s). Publication rights licensed to ACM ACM ISBN 978-1-4503-8969-3/21/05.

https://doi.org/10.1145/3468691.3468731
Internet of Things (CNIOT 2021) (CNIOT2021), May 20-22, 2021, Beijing, China. ACM, New York, NY, USA, 7 pages. https://doi.org/10.1145/3468691.3468731

\section{INTRODUCTION}

GQN (Generative Query Network) [2] is one of the generative models proposed by DeepMind. It manages to learn the representation of a 3D structure, which paves the way to the scene understanding and rendering. The intuition behind this model is that given several 2D projections (contexts) of a 3D object with their corresponding viewpoints, our brain is able to have a representation of this object, and perspectives from other viewpoints can be imagined in our mind. GQN uses convolutional neural networks[13] to obtain the context representation and Convolutional LSTM[5] to make the prediction.

However, training GQN usually takes hours and even days on a single GPU, due to a large number of weights to be updated. Model Parallelism and Data Parallelism[11] are the two common methodologies to accelerate the process in distributed environment but in practice, given high model complexity, communication overhead is no longer negligible and may impede performance scalability on even small to medium scale clusters. It is of great importance to accelerate the training process of GQN for its fast deployment and to that end, we propose a two-phased model which greatly reduces the model complexity. It contains two modules. One is Structure Model,which captures the structural features of the observed geometry in gray-scale image and predicts gray-scale perspectives according to queried viewpoints. The intermediate result is then fed to the Colorization Network, which repaints the perspective based on the original context. In this approach, the number of parameters is greatly reduced. We also apply the gradient accumulation technique and avoid redundant gradient broadcast and thus alleviate extra communication overhead. 


\section{RELATED WORK}

\subsection{GQN principles [2]}

GQN is originated from VRNN (recurrent latent variational model [1]). Benefit from the power of VRNN in modeling highly structured data, GQN succeeds in generating effective representation of sequences of images. There are three modules in GQN, the representation network, the generation network and the inference network.

2.1.1 Representation Network. The representation network is a function that transforms context images and their corresponding viewpoints into a vector. In detail, each context image and its viewpoint vector is fed into a shared residual convolutional network to obtain their vector representation respectively. Then, these vectors will be summed to gain the vector of the representation of the whole scene.

2.1.2 Generation Network. The generation network has two functions. First, it produces the required image according to the scene representation and the query viewpoint. Second, it maps them to sufficient statistics of the distribution (i.e. mean and variance for normal distribution) of the sequential latent variables. For latent variable models, we call the distribution "prior", relative to the distribution depicted in inference network. The backbone of generation network is convolutional LSTM.

2.1.3 Inference Network. The posterior distribution of latent variables is also inferred, and this is done by the Inference Network. This distribution is approximated by both of the scene representation with the query viewpoint and ground truth. The loss function of GQN is ELBO(evidence lower bound), which is the sum of negative log likelihood of the ground truth image and the kl-divergence between the prior and the posterior stated above.

\subsection{Reference GQN implementation}

Since there is no official implementation published by DeepMind, it is a common practice that further research on GQN are based on open-source implementations [16]. This implementation is pytorchbased and is proved to produce similar results as the original work[2].

Pytorch is an open-source machine learning framework based on Python. It comes with a simple interface and supports CUDA to speed up training with GPUs. In Pytorch, the way to perform distributed training is Data Parallelism, which implemented as DP (Data Parallel) and DDP (Distributed Data Parallel)[8]. DP is designed to deploy multi-GPUs training in a single host with a master-slave architecture and the imbalance of node load makes it difficult to expand training scale. In comparison, DDP is fully distributed among training processes and uses collective communications (i.e. AllReduce[10]) to efficiently synchronize gradients and buffers among hosts, so it is possible to perform large-scale training with DDP. Interested readers can refer to [7] for detail.

\subsection{Image Colorization}

In our approach, the inputs and outputs of structure model are single channel images needed coloring. There are abundant research in image colorization. Zhang et al. [17] trains a discriminative model to classify categorized colors with convolutional encoder-decoder.
Other methods are inspired by generative adversarial training, such as pix2pix[4], pix2pixHD[14] and cycleGAN[18]. However, previous works on auto-rendering are not directly applicable in our approach because they neglect the context color information and may introduce redundant computation in the specific use of GQN acceleration.

\section{OUR APPROACH}

\subsection{Train generation network with Context-aware Colorization(2P-GQN)}

3.1.1 Basic Assumptions And Ideas. The target that we want to maximize is $p_{\Theta}\left(\mathbf{x}^{q} \mid \mathbf{v}^{q}, \mathbf{r}\right)$, which is the likelihood of image $\mathbf{x}^{q}$ given a certain viewpoint $\mathbf{v}^{q}$ and the context representation $\mathrm{r}$, parameterized by $\Theta$. There are three channels in one image $\mathrm{x}^{q}$. In CIE-LAB colorspace, these channels are lightness $l, a$ value from green to red, and $b$ value from blue to yellow. Denote $\mathbf{x}^{q}=\left(\mathbf{l}^{q}, \mathbf{a}^{q}, \mathbf{b}^{q}\right)$, we have following equations $\left(\Theta=\theta_{1} \cup \theta_{2}\right)$.

$$
p_{\Theta}\left(\mathbf{l}^{q}, \mathbf{a}^{q}, \mathbf{b}^{q} \mid \mathbf{v}^{q}, \mathbf{r}\right)=p_{\theta_{1} \in \Theta}\left(\mathbf{l}^{q} \mid \mathbf{v}^{q}, \mathbf{r}\right) * p_{\theta_{2} \in \Theta}\left(\mathbf{a}^{q}, \mathbf{b}^{q} \mid \mathbf{v}^{q}, \mathbf{l}^{q}, \mathbf{r}\right)
$$

We provide two assumptions to make the optimization problem simpler.

Assumption 1. The single channel image $\mathbf{1}^{q}$ is independent of $a$ channel and $b$ channel in context images. That is:

$$
p_{\theta_{1}}\left(\mathbf{l}^{q} \mid \mathbf{v}^{q}, \mathbf{r}\right)=p_{\theta_{1}}\left(\mathbf{l}^{q} \mid \mathbf{v}^{q}, \mathbf{r}^{\prime}\right)
$$

Here, $\mathbf{r}^{\prime}=f^{\prime}\left(\mathbf{l}_{1}, \mathbf{l}_{2}, \ldots, \mathbf{l}_{m}, \mathbf{v}_{1}, \mathbf{v}_{2}, \ldots, \mathbf{v}_{m}\right) . \mathbf{l}_{1}, \mathbf{l}_{2}, \ldots, \mathbf{l}_{m}$ represents the $\mathrm{L}$ channel of $m$ context images.

Assumption 2. $\theta_{1}$ and $\theta_{2}$ is a partition of $\Theta$. That means, $\theta_{1} \cap \theta_{2}=$ $\Phi$, which leads to:

$$
\max _{\Theta} p_{\Theta}\left(1^{q}, \mathbf{a}^{q}, \mathbf{b}^{q} \mid \mathbf{v}^{q}, \mathbf{r}\right)=\max _{\theta_{1}} p_{\theta_{1}}\left(\mathbf{l}^{q} \mid \mathbf{v}^{q}, \mathbf{r}\right) * \max _{\theta_{2}} p_{\theta_{2}}\left(\mathbf{a}^{q}, \mathbf{b}^{q} \mid \mathbf{v}^{q}, 1^{q}, \mathbf{r}\right)
$$

Considering (2) and (3), we have:

$$
\max _{\Theta} p_{\Theta}\left(\mathbf{l}^{q}, \mathbf{a}^{q}, \mathbf{b}^{q} \mid \mathbf{v}^{q}, \mathbf{r}\right)=\max _{\theta_{1}} p_{\theta_{1}}\left(\mathbf{l}^{q} \mid \mathbf{v}^{q}, \mathbf{r}^{\prime}\right) * \max _{\theta_{2}} p_{\theta_{2}}\left(\mathbf{a}^{q}, \mathbf{b}^{q} \mid \mathbf{v}^{q}, \mathbf{l}^{q}, \mathbf{r}\right)
$$

Based on these two assumptions, we make improvement on traditional GQN model, breaking it down into two modules, including one for structure-prediction and another for colorization. The structure-prediction model predicts only one channel of the target image(e.g. light channel), and the colorization model is responsible for pixel values of all other channels. Compared with end-to-end training, this method has two advantages. To get the same performance, the number of parameters in the structure model is reduced compared with traditional GQN. For the colorization model, only $10 \%$ volume of data will make converge to the optima. Benefited from these two factors, the overall training speed has improved greatly.

3.1.2 The Structure Model. For dataset $\mathcal{D}=\left\{\mathbf{x}_{i}, \mathbf{v}_{i}\right\}_{i=1}^{N}$, each sample is a sequence of $\mathrm{K}$ images with height $H$ and width $W$. That is $\mathbf{x}_{i}=\mathbf{x}_{i}^{1}, \mathbf{x}_{i}^{2}, \ldots, \mathbf{x}_{i}^{K}$ and $\mathbf{v}_{i}=\mathbf{v}_{i}^{1}, \mathbf{v}_{i}^{2}, \ldots, \mathbf{v}_{i}^{K}$. To train our model, we have to partition the dataset into two parts with respect to the time step dimension, leaving out the query step, say $\mathbf{x}_{i}=\left\{\mathbf{x}_{i}^{1}, \mathbf{x}_{i}^{2}, \ldots, \mathbf{x}_{i}^{M}\right\} \cup\left\{\mathbf{x}_{i}^{q}\right\}$ and $\mathbf{v}_{i}=\left\{\mathbf{v}_{i}^{1}, \mathbf{v}_{i}^{2}, \ldots, \mathbf{v}_{i}^{M}\right\} \cup\left\{\mathbf{v}_{i}^{q}\right\}$, here $M=K-1$. (For training, we apply same function to all elements of one minibatch, therefore, superscripts will be omitted below 


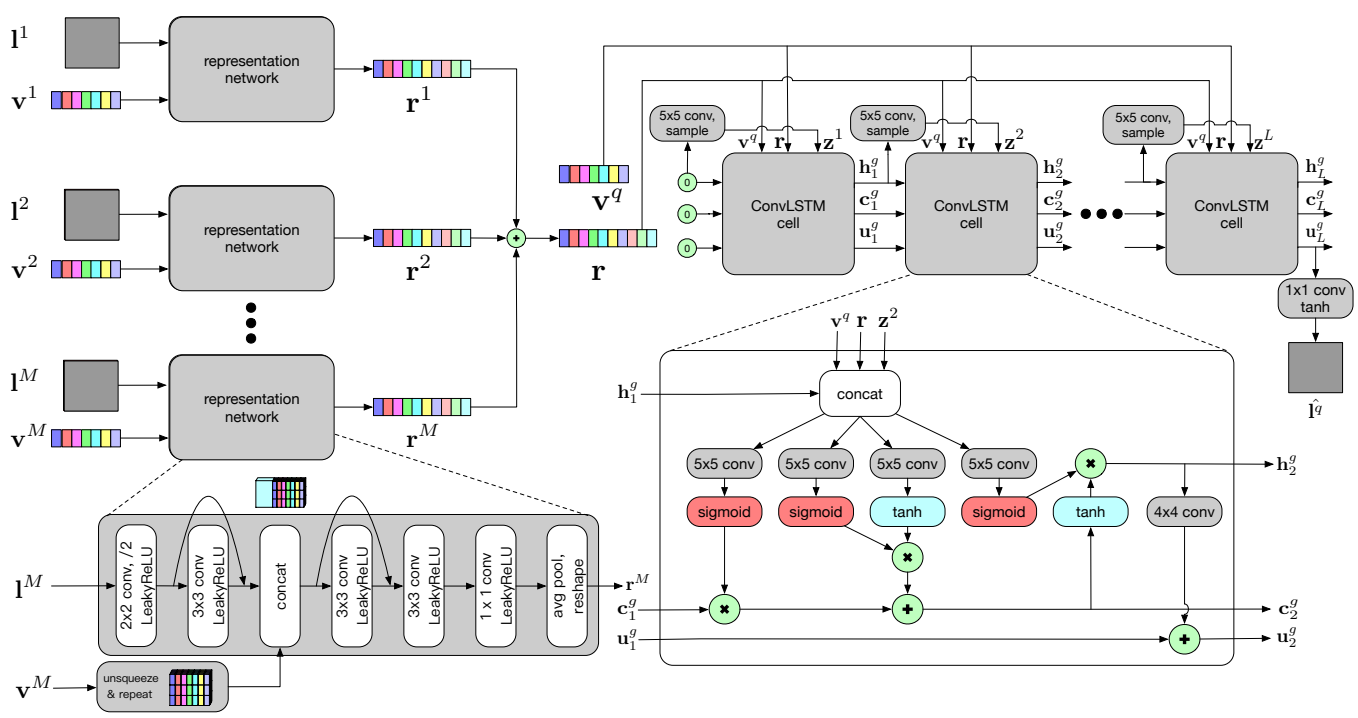

Figure 1: The Architecture of Structure Model

if no ambiguity happens.) Our structure model only takes onedimensional image as input. For image in CIE-LAB colorspace $\mathbf{x}=[\mathbf{l}, \mathbf{a}, \mathbf{b}]$, we only input $\mathbf{l} \in \mathcal{R}^{1 \times H \times W}$.

The structure model includes representation network, generation network and inference network. The representation network is able to encode those one-channel context images into a vector $\mathbf{r}$. The generation network is capable of modeling probability densities of required image $g_{\phi_{1}}\left(\mathbf{l}^{q} \mid \mathbf{z}, \mathbf{v}^{q}, \mathbf{r}\right)$ and the prior of latent variables $\pi_{\phi_{2}}\left(\mathbf{z} \mid \mathbf{v}^{q}, \mathbf{r}\right)$. The inference network parameterizes the posterior distribution of latent variable $q_{\phi_{3}}\left(\mathbf{z} \mid 1^{q}, \mathbf{v} q, \mathbf{r}\right)$. Obviously, $\theta_{1}=\left(\phi_{1}, \phi_{2}, \phi_{3}\right)$.

Representation Network. A shared representation network encodes $M$ one-channel images and their viewpoint vectors with convolution and pooling layers. Detail information about the representation network is on 1 .

$$
\mathbf{r}=\sum_{k=1}^{M} \operatorname{ConvNet}_{\phi_{1}}\left(\mathbf{l}^{\mathrm{k}}, \mathbf{v}^{\mathrm{k}}\right)
$$

Generation Network. According to figure 1, the backbone of generation network is convolutional LSTM. The one-channel image to predict is derived by following equations:

$$
\begin{aligned}
\left(\mathbf{h}_{0}^{g}, \mathbf{c}_{0}^{g}, \mathbf{u}_{0}^{g}\right) & =(\mathbf{0}, \mathbf{0}, \mathbf{0}) \\
\pi_{\phi_{1}}\left(\cdot \mid \mathbf{v}^{q}, \mathbf{r}, \mathbf{z}_{<l}\right) & =\mathcal{N}\left(\cdot \mid C_{\phi_{1}}^{g}\left(\mathbf{h}_{l}^{g}\right)\right) \\
\mathbf{z}_{l}^{g} & \sim \pi_{\phi_{1}}\left(\cdot \mid \mathbf{v}^{q}, \mathbf{r}, \mathbf{z}_{<l}\right) \\
\left(\mathbf{h}_{l+1}^{g}, \mathbf{c}_{l+1}^{g}, \mathbf{u}_{l+1}^{g}\right) & =\operatorname{ConvLSTM}_{\phi_{1}}\left(\mathbf{h}_{1}^{\mathrm{g}}, \mathbf{c}_{1}^{\mathrm{g}}, \mathbf{u}_{1}^{\mathrm{g}}, \mathbf{z}_{1}^{\mathrm{g}}, \mathbf{v}^{\mathrm{q}}, \mathbf{r}\right) \\
\hat{\mathbf{l}^{q}} \sim \mathcal{N}(\cdot \mid \mu & \left.=\mathrm{C}_{\phi_{1}}^{\mathrm{g}^{\prime}}\left(\mathbf{u}_{\mathrm{L}}\right), \sigma=\sigma_{\mathrm{t}}\right)
\end{aligned}
$$

The convolutional layer $C_{\phi_{1}}^{g}$ maps the hidden state of time step $l$ to sufficient statistics of gaussian variational prior density of the latent variable(mean and variance). Then we sample latent variable from this prior density and feed it into convolutional LSTM cell with previous states, cell values, the query viewpoint vector and the representation vector. The vector which represents required image is $\mathbf{u}_{L}$, and $C_{\phi_{1}}^{g^{\prime}}$ transforms $\mathbf{u}_{L}$ into mean value of $\hat{\imath} \hat{q}$. For its standard deviation, we use linear per-pixel standard deviation annealing. That is:

$$
\sigma_{t}=\max \left(\sigma_{f}+\left(\sigma_{0}-\sigma_{f}\right)\left(1-\frac{t}{T}\right), \sigma_{f}\right) \quad\left(\sigma_{0}>\sigma_{f}\right)
$$

The standard deviation will be decayed from $\sigma_{0}$ to $\sigma_{f}$ in $T$ time steps.

Inference Network. The goal of inference network is to model the posterior distribution of the latent variable. The posterior is expressed by equations below:

$$
\begin{aligned}
\left(\mathbf{h}_{0}^{e}, \mathbf{c}_{0}^{e}\right) & =(\mathbf{0}, \mathbf{0}) \\
\left(\mathbf{h}_{l+1}^{e}, \mathbf{c}_{l+1}^{e}\right) & =\operatorname{ConvLSTM} \phi_{\phi_{2}}\left(\mathbf{h}_{1}^{\mathrm{g}}, \mathbf{h}_{1}^{\mathrm{e}}, \mathbf{c}_{1}^{\mathrm{e}}, \mathbf{u}_{1}, \mathbf{1}^{\mathrm{q}}, \mathbf{v}^{\mathrm{q}}, \mathbf{r}\right) \\
q_{\phi_{2}}\left(\cdot \mid \mathbf{x}^{q}, \mathbf{v}^{q}, \mathbf{r}, \mathbf{z}_{<l}\right) & =\mathcal{N}\left(\cdot \mid C_{\phi_{2}}^{e}\left(\mathbf{h}_{l}^{e}\right)\right)
\end{aligned}
$$

The structure of ConvLSTM $\phi_{2}$ is much similar to ConvLSTM $\phi_{1}$ in 1 , but the input into the concatenate operation at time step $l+1$ is $\mathbf{h}_{l}^{g}, \mathbf{l}^{q}, \mathbf{v}^{\mathbf{q}}$ and $\mathbf{r}$. The convolutional LSTM in inference network does not share parameters with the one in generation network.

Optimization Goal. To train generation network, our optimization goal is the elbo loss, which contains negative log likelihood of ground truth and the KL-divergence of latent prior and posterior.

$$
\begin{aligned}
L\left(\theta_{1}\right)= & E_{(\mathbf{x}, \mathbf{v}) \sim D}\left[-\ln \mathcal{N}\left(\mathbf{l}^{q} \mid C_{\phi_{1}}^{g^{\prime}}\left(\mathbf{u}_{L}\right)\right)\right] \\
& +E_{(\mathbf{x}, \mathbf{v}) \sim D} \sum_{l=1}^{L} \mathrm{KL}\left[\mathcal{N}\left(\cdot \mid \mathrm{C}_{\phi_{1}}^{\mathrm{g}}\left(\mathbf{h}_{1}^{\mathrm{g}}\right)\right)|| \mathcal{N}\left(\cdot \mid \mathrm{C}_{\phi_{2}}^{\mathrm{e}}\left(\mathbf{h}_{1}^{\mathrm{e}}\right)\right)\right]
\end{aligned}
$$

3.1.3 The Colorization Model. We define the context-aware colorization as a mapping from context colorized images $\mathbf{x}_{1}, \mathbf{x}_{2}, \ldots, \mathbf{x}_{M}$, 
their viewpoints $\mathbf{v}_{1}, \mathbf{v}_{2}, \ldots, \mathbf{v}_{M}$, plus the output of structure model $\mathbf{l}^{q}$, to the rendered $\hat{I}^{q}=\left(\hat{\mathbf{a}_{q}}, \hat{\mathbf{b}_{q}}\right)$, which represents the other two channels required to output. Although this problem can solved by another CVAE, we finally choose vanilla autoencoder with some modification, because it introduces the least additional parameters and computation. To get final image $\hat{\mathbf{x}^{q}}$, we make a channel-wise concatenation of $\mathbf{l}_{q}$ and $\hat{\mathrm{I}} \boldsymbol{q}$ to get the final output. Here is how to get $\hat{\mathbf{I}}$.

$$
\hat{\mathbf{I}} \underline{q}=Q_{\theta_{2}}\left(\mathbf{x}_{1}, \mathbf{x}_{2}, \ldots, \mathbf{x}_{M}, \mathbf{v}_{1}, \mathbf{v}_{2}, \ldots, \mathbf{v}_{M}, \hat{\mathbf{l}}, \mathbf{v}^{q}\right)
$$

The function $Q$ consists of four parts: context encoder $g\left(\lambda_{1}\right)$, query encoder $h\left(\lambda_{2}\right)$, fusion layer $F\left(\lambda_{3}\right)$ and decoder $G(\xi)$. So, $\theta_{2}=\left\{\lambda_{1}, \lambda_{2}, \lambda_{3}, \xi\right\}$. The context encoder transforms three-channel context images $\mathbf{x}_{1}, \mathbf{x}_{2}, \ldots, \mathbf{x}_{M}$ (in CIE-LAB colorspace) and context viewpoints $\mathbf{v}_{1}, \mathbf{v}_{2}, \ldots, \mathbf{v}_{M}$ to the corresponding representation $r_{1}$ Meanwhile, the query encoder maps the 1-channel image output by structure model to query representation $\mathbf{r}_{2}$. Then, $\mathbf{r}_{1}$ and $\mathbf{r}_{2}$ will be fed into the fusion layer $F$, producing vector $\mathbf{r}$, the hidden representation of $\hat{\mathbf{I} q}$.

$$
\begin{aligned}
\mathbf{r}_{1} & =\sum_{k=1}^{M} g_{\lambda_{1}}\left(\mathbf{x}_{i}, \mathbf{v}_{i}\right) \\
\mathbf{r}_{2} & =h_{\lambda_{2}}\left(\hat{\mathbf{l}}, \mathbf{v}^{q}\right) \\
\mathbf{r} & =\mathrm{F}_{\lambda_{3}}\left(\mathbf{r}_{1}, \mathbf{r}_{2}\right) \\
\mathbf{I}^{\hat{q}} & =\tanh \left(G_{\xi}(\mathbf{r})\right)
\end{aligned}
$$

The structure of $g_{\lambda_{1}}$ and $h_{\lambda_{2}}$ is the same as the structure network. The parameter $\lambda_{1}$ is shared across $M$ context images, but not with those in structure model. Suppose we have $M$ context images $\left\{\mathbf{x}_{k} \in \mathbb{R}^{3 \times H \times W}\right\}_{k=1}^{M}$ and viewpoints $\left\{\mathbf{v}_{k} \in \mathbb{R}^{V}\right\}_{k=1}^{M}$, the context encoder will output $\mathbf{r}_{1} \in \mathbb{R}^{r c}$ and the query encoder will output $\mathbf{r}_{2} \in \mathbb{R}^{r q}$. These two representations will be input into fusion layer, producing vector $\mathbf{r} \in \mathbb{R}^{\text {hidden }}$. In this layer, two representations will be concatenated first, then going through a fully connected projection layer with activation function $\sigma$ (Leaky ReLU here).

$$
\mathbf{r}=\sigma\left(\mathrm{fc}_{\lambda_{3}}\left(\left[\mathbf{r}_{1} ; \mathbf{r}_{2}\right]\right)\right)
$$

Finally, the representation will be decoded into the predicted image. The decoder network $\mathrm{G}$ composes of 1 reshape operation, 4 transpose convolution layers with LeakyReLU activation except the last layer (see 2). The feature map is upsampled until it reaches the target shape. Because $l^{q}$ has already given, we just need to predict other two channels, $\hat{a}^{q}$ and $\hat{b^{q}}$. We use tanh function to squeeze the output between $(-1,1)$. For dataset with $N$ samples, the concatenation of $a$ channel and $b$ channel of ground truth image of scene $i$ is denoted as $I_{i}^{q}$. The loss function is:

$$
L\left(\theta_{2}\right)=\frac{1}{2 N} \sum_{i=1}^{N}\left\|\mathbf{I}_{i}{ }^{q}-\hat{\mathbf{I}}_{i}^{q}\right\|_{2}^{2}
$$

3.1.4 Compare 2P-GQN with traditional GQN. We make a comparison between traditional GQN and our 2P-GQN. From the table, we see that in $2 \mathrm{P}-\mathrm{GQN}$ model, the total number of parameters is reduced by $45.6 \%$ even though we have two models in $2 \mathrm{P}-\mathrm{GQN}$.

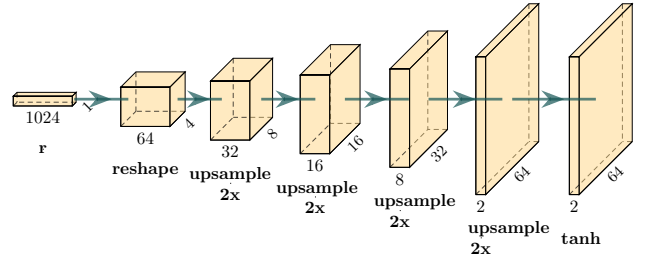

Figure 2: The structure of decoder G

Table 1: Parameter Comparison Between GQN and 2P-GQN

\begin{tabular}{ccl}
\hline Item & GQN & 2P-GQN \\
\hline Input channels & 3 & 1 \\
Dimension of $\mathbf{r}$ & 256 & 128 \\
Dimension of $\mathbf{h}$ & 128 & 64 \\
Dimension of $\mathbf{z}$ & 64 & 64 \\
Parameters in generator & 13.19 Million & 3.81 Million \\
Parameters in colorization model & - & 3.36 Million \\
Total parameters & 13.19 Million & 7.17 Million \\
\hline
\end{tabular}

\subsection{Gradient Accumulation[8] and Communication Overhead Alleviation}

Gradient accumulation is a trick to further increase batch size when training without enough hardware resources, and larger batch size, in a way, is beneficial to expedite the training process[9]. By default, DDP training performs all-reduce operation implicitly during backward propagation, causing a lot of communication overhead.

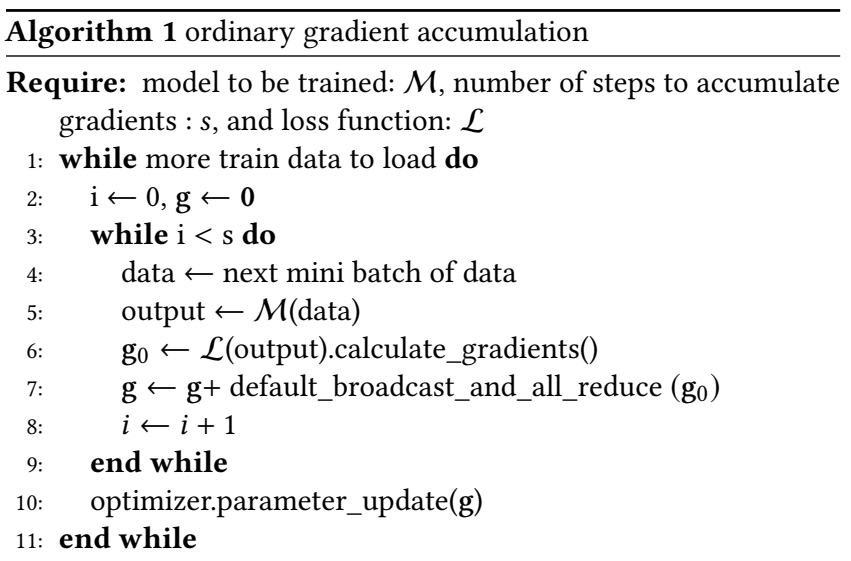

In this paper, we explicitly disable gradient propagation and synchronization during gradient accumulation. The algorithm is stated in 2. 


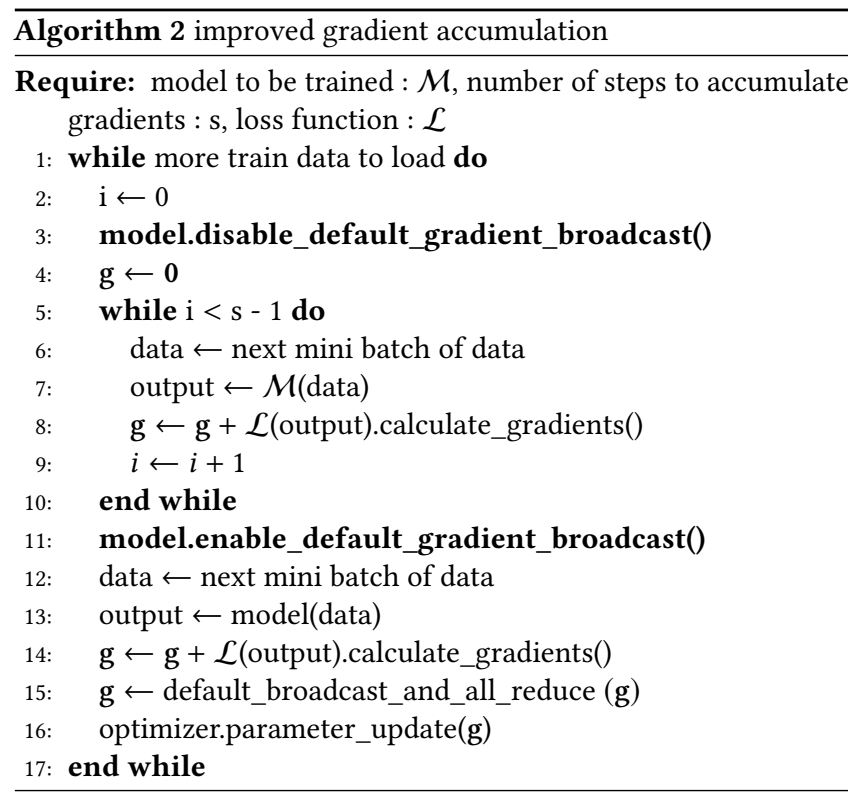

As a result, we managed to reduce $s-1$ times of communication if we set accumulation steps to $s$.

\section{EXPERIMENTS}

\subsection{Experimental settings}

We use Shepard-Metzler, which is a mental rotation dataset. The task was first proposed in [12] and the dataset was created by DeepMind.

In our experiments, the server has one i7-7820X CPU, 4 Nvidia GTX Titan Xp GPUs (with 12GB memory) and 64GB RAM. The operating system is CentOS 7 with CUDA 9.2 installed. To parallelize our model, we use DistributedDataParallel in PyTorch with "gloo" backend.

\subsection{Train 2P-GQN}

The structure model and colorization model are trained separately. To show the training speed improvement of our method, we make a comparison with traditional GQN model.

4.2.1 Context Aware Colorization Model. The colorization model is trained with batch size 64 and Adam optimizer[6] with learning rate $10^{-3}$. We use only $10 \%$ of the mental rotation dataset to train our colorization model.

4.2.2 Structure Model. The structure model is trained with batch size 64 . We use Adam optimizer with learning rate $3 * 10^{-4}$. To make the training process fully converged, we go over the dataset 50 epochs. We validate our model on the validation set after every epoch of training. During validation, we record the model performance and training time every epoch.

4.2.3 Prediction Examples. In figure 3, 14 context images containing different viewpoints of one certain object are given.

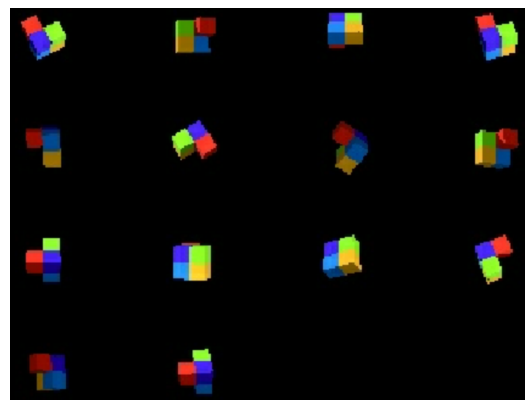

Figure 3: 14 context images

Figure 4 shows that we take the output of structure model, grey image, as the query input of the colorization model. Combined with those context images, we get the output, colored image, as the prediction which is quite close to the ground truth.

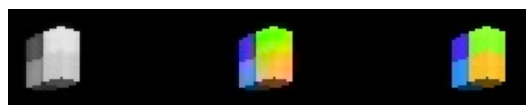

Figure 4: The input of colorization network(left), the prediction of the colorization model (middle) and the ground truth(right)

4.2.4 Evaluation Metric. Our evaluation metric of generated image quality is mean structural similarity(SSIM) [15] of generated image and the ground truth across the validation set.

Here, we choose $L=255, \alpha=\beta=\gamma=1, C_{1}=0.01 \cdot L^{2}$, $C_{2}=0.03 \cdot L^{2}$ and $C_{3}=C_{2} / 2$, according to common practices. In our experiment, colorized images are output in CIE-LAB color space in range $[-1,1]$. We scale this output linearly to range $[0,255]$ and input each channel into SSIM function with corresponding channel in ground truth image. Then, we average these similarity values to get the final similarity.

4.2.5 Overall Performance Comparison. To show the effectiveness of two-phase training, we make a comparison between traditional GQN and 2P-GQN on the structural similarity over time.

Since we train two models in $2 \mathrm{P}-\mathrm{GQN}$, to make a fair comparison, both of structure model and colorization model are run 50 epochs. The recorded training time of epoch $t$ in 2P-GQN is the sum of training time of structure model and colorization model of epoch $t$.

From 5, we can see that $2 \mathrm{P}-\mathrm{GQN}$ reduces the training time by $56.92 \%$ at a cost of 0.016 structural similarity less than the traditional one, when both of these two models are trained for 50 epochs. To gain 0.907 SSIM, the training time of traditional GQN is 35698.3 seconds as compared to 23305.7 seconds of $2 \mathrm{P}-\mathrm{GQN}$. Therefore, to achieve the same performance (we record validation structural similarity one time per epoch, so we don't have data points with SSIM 0.908), the training time is reduced by $34.7 \%$ at least.

\subsection{Improved Gradient Accumulation(IGA)}

We separately trained the IGA and original GQN model in the same environment and record the time required to reach the convergence whose criterion is that the loss(ELBO) no longer changes drastically. 

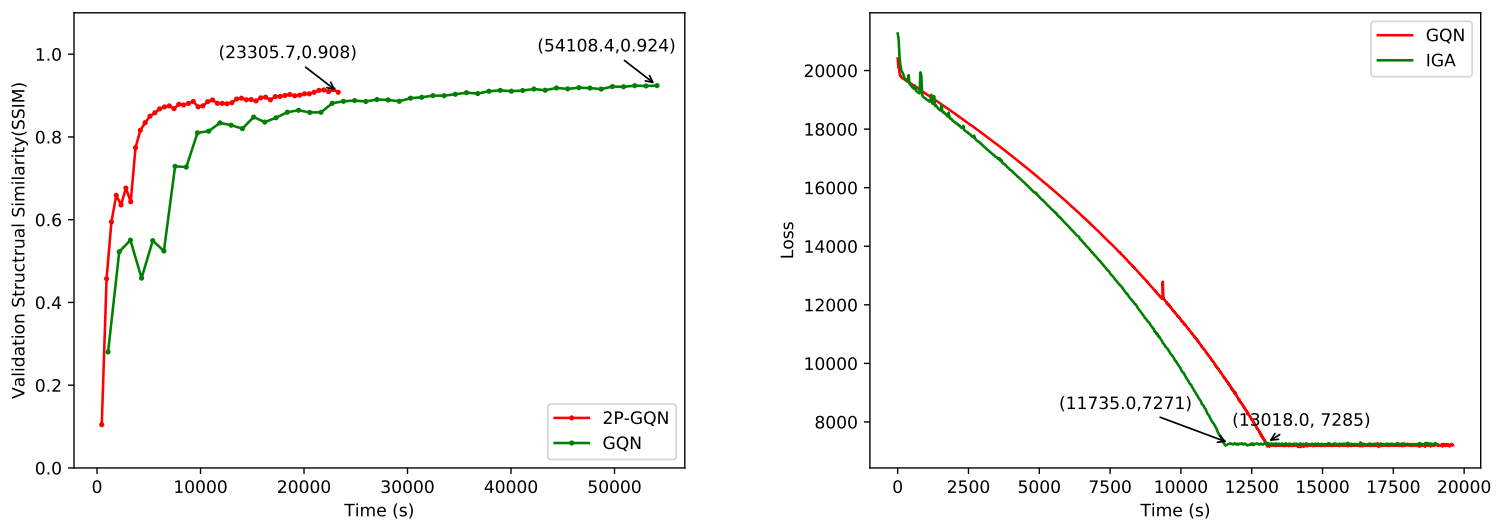

Figure 5: (left)Performance Comparison between 2P-GQN and traditional GQN. (right)Time comparison between IGA and GQN

4.3.1 Experiment Environment. The batch size for the two experiments is 64. For the original GQN, we use the Adam optimizer with learning rate $3 * 10^{-4}$. However, considering that for the IGA, we accumulate 4 iterations of gradients before one update, so we should enlarge the value of learning rate. We follow the Linear Scaling Rule [3], setting the learning rate to $1.2 * 10^{-3}$, and we go over 50 epochs to ensure we reach the final convergence.

4.3.2 Performance Comparison. To show the effectiveness of IGA, we make a comparison between traditional GQN and IGA on the training loss over time.

As the experiments show, the IGA's and GQN's loss converge to similar value(about 7000) and under the same environmental conditions, IGA takes shorter time to converge, which is about $9.86 \%$ speedup. Since in DDP, the gain of IGA is enlarged batch size with less communication overhead, when we train the model with more machines, this acceleration effect will be more significant.

\section{CONCLUSION}

GQN is a novel generative model based on VRNN, which has various interesting applications in the field of computer vision. It has a massive amount of parameters and it is time-consuming to train a useful GQN network in practice. For a faster deployment of GQN on typical dense GPGPU platform, optimizations to the original GQN are proposed in this paper. We simplify the GQN model by omitting the color information in the structural perception phase and repaint the image in colorization phase. Afterwards, we also apply gradient accumulation techniques and further reduce the communication overhead. And experimental results show that, without significant loss of quality of the generated images, we reduce $56.92 \%$ training time by two-phase training and $9.86 \%$ training time by improved gradient accumulation. In the future, we intend to test our approach on large-scale heterogeneous GPU clusters and try to apply our approach in other large neural rendering models.

\section{ACKNOWLEDGMENTS}

This paper is sponsored by Youth Innovation Promotion Association CAS.

\section{REFERENCES}

[1] J. Chung, Kyle Kastner, Laurent Dinh, Kratarth Goel, Aaron C. Courville, and Yoshua Bengio. 2015. A Recurrent Latent Variable Model for Sequential Data. In NIPS.

[2] S. M. Ali Eslami, Danilo Jimenez Rezende, Frederic Besse, Fabio Viola, Ari S. Morcos, Marta Garnelo, Avraham Ruderman, Andrei A. Rusu, Ivo Danihelka, Karol Gregor, David P. Reichert, Lars Buesing, Theophane Weber, Oriol Vinyals, Dan Rosenbaum, Neil Rabinowitz, Helen King, Chloe Hillier, Matt Botvinick, Daan Wierstra, Koray Kavukcuoglu, and Demis Hassabis. 2018. Neural scene representation and rendering. Science 360, 6394 (2018), 1204-1210. https://doi.org/10.1126/science.aar6170 arXiv:https://science.sciencemag.org/content/360/6394/1204.full.pdf

[3] Priya Goyal, Piotr Dollár, Ross Girshick, Pieter Noordhuis, Lukasz Wesolowski, Aapo Kyrola, Andrew Tulloch, Yangqing Jia, and Kaiming He. 2017. Accurate, Large Minibatch SGD: Training ImageNet in 1 Hour. (2017).

[4] Phillip Isola, Jun-Yan Zhu, Tinghui Zhou, and Alexei A. Efros. 2017. Image-toImage Translation with Conditional Adversarial Networks. 2017 IEEE Conference on Computer Vision and Pattern Recognition (CVPR) (2017), 5967-5976.

[5] Seongchan Kim, Seungkyun Hong, Minsu Joh, and Sa-Kwang Song. 2017. DeepRain: ConvLSTM Network for Precipitation Prediction using Multichannel Radar Data. CoRR abs/1711.02316 (2017). arXiv:1711.02316 http://arxiv.org/abs/1711. 02316

[6] Diederik Kingma and Jimmy Ba. 2014. Adam: A Method for Stochastic Optimization. Computer ence (2014).

[7] Shen Li. [n.d.]. GETTING STARTED WITH DISTRIBUTED DATA PARALLEL. https://pytorch.org/tutorials/beginner/dist_overview.html.

[8] Shen Li, Yanli Zhao, Rohan Varma, Omkar Salpekar, Pieter Noordhuis, Teng Li, Adam Paszke, Jeff Smith, Brian Vaughan, Pritam Damania, and Soumith Chintala. 2020. PyTorch Distributed: Experiences on Accelerating Data Parallel Training. (06 2020).

[9] Myle Ott, Sergey Edunov, David Grangier, and Michael Auli. 2018. Scaling Neural Machine Translation. CoRR abs/1806.00187 (2018). arXiv:1806.00187 http://arxiv.org/abs/1806.00187

[10] Pitch Patarasuk and Xin Yuan. 2009. Bandwidth optimal all-reduce algorithms for clusters of workstations. F. Parallel and Distrib. Comput. 69, 2 (2009), 117 124. https://doi.org/10.1016/j.jpdc.2008.09.002

[11] Christopher J. Shallue, Jaehoon Lee, Joseph M. Antognini, Jascha Sohl-Dickstein, Roy Frostig, and George E. Dahl. 2018. Measuring the Effects of Data Parallelism on Neural Network Training. CoRR abs/1811.03600 (2018). arXiv:1811.03600 http://arxiv.org/abs/1811.03600

[12] Roger N Shepard and Jacqueline Metzler. 1971. Mental rotation of threedimensional objects. Science 171, 3972 (1971), 701-703. 
[13] Kihyuk Sohn, Xinchen Yan, Honglak Lee, and Ann Arbor. 2015. Learning Structured Output Representation using Deep Conditional Generative Models. In International Conference on Neural Information Processing Systems.

[14] T. Wang, Ming-Yu Liu, Jun-Yan Zhu, A. Tao, J. Kautz, and Bryan Catanzaro. 2018 High-Resolution Image Synthesis and Semantic Manipulation with Conditional GANs. 2018 IEEE/CVF Conference on Computer Vision and Pattern Recognition (2018), 8798-8807.

[15] Zhou Wang, A. Bovik, H. R. Sheikh, and E. P. Simoncelli. 2004. Image quality assessment: from error visibility to structural similarity. IEEE Transactions on
Image Processing 13 (2004), 600-612.

[16] Jesper Wohlert. [n.d.]. generative-query-network-pytorch. https://github.com/ wohlert/generative-query-network-pytorch.

[17] Richard Zhang, Phillip Isola, and Alexei A. Efros. 2016. Colorful Image Colorization. In $E C C V$.

[18] Jun-Yan Zhu, T. Park, Phillip Isola, and Alexei A. Efros. 2017. Unpaired Imageto-Image Translation Using Cycle-Consistent Adversarial Networks. 2017 IEEE International Conference on Computer Vision (ICCV) (2017), 2242-2251. 